\title{
ON THE DIGITISATION OF ODESSA COLLECTION OF ASTRONOMICAL NEGATIVES. EXAMINATION OF THE EPSON PERFECTION V700 PHOTO SCANNER
}

\author{
S. Kashuba ${ }^{1}$, V. Andruk ${ }^{2}$, V. Kashuba ${ }^{1}$ \\ ${ }^{1}$ Astronomical Observatory of I. I. Mechnikov Odessa National University, \\ Shevchenko park, Odessa 65014, Ukraine,sv-k@onu.edu.ua \\ ${ }^{2}$ Main Astronomical Observatory of the National Academy of Sciences of Ukraine, \\ Akad. Zabolotnoho St.27, Kyiv 03680, Ukraine, andruk1058@ukr.net
}

\begin{abstract}
This study was aimed to estimate the precision of the selected plate processing method and test the accuracy of the Epson Perfection V700 Photo scanner used to digitize astroplates from the Odessa Astronomical Observatory's collection. The processing of astroplates was performed using specific software developed in the LINUX/MIDAS/ROMAFOT environment. In order to estimate the repeatability of the scanner's astrometric and photometric errors, six consecutive scans of one and the same plate (No 17,000 the Pleiades star cluster) were processed in two scanning modes with spatial resolutions of 1,200 and 2,400 dpi. The plate was exposed for 30 minutes with the fourth tube of the Seven Wide Angle (SWA) astrograph. The scan size was $18 \times 24 \mathrm{~cm}$ (13 x 18 degrees). The following estimates of the scanner's accuracy were obtained for the stars brighter than $\mathrm{B}<13 \mathrm{~m}$ in the two scanning modes: 1) $\sigma_{x y}= \pm 0.023 \mathrm{px}, \sigma_{m}= \pm 0.013^{\mathrm{m}}$; 2) $\sigma_{x y}= \pm 0.082 \mathrm{px}$, $\sigma_{m}= \pm 0.042^{\mathrm{m}}$ for the Cartesian coordinates and instrumental magnitude, respectively.
\end{abstract}

Keywords: astronegative, scanning, processing of digitized plates

\section{Introduction}

In the Odessa Astronomical Observatory they intend to digitise a unique collection of astronomical photographic plates amassed over many years of systematised patrol observations (Vavilova, 2011). The earliest plate is dated 1909, and the total number of plates exceeds 110 thousand. The collection has been acquired with various instruments and covers most of the northern hemisphere. Thus far, test scanning of several hundred of plates has been performed using Epson Perfection V700 Photo in order to assess the quality of scanning and suitability of digitised images for setting various research objectives.

\section{Scanning results at 1200 and $2400 \mathrm{dpi}$}

In this study aimed at testing the accuracy of the Epson Perfection V700 Photo scanner, the authors used a method which has already been employed earlier in a number of works (Golovnya, 2010; Eglitis, 2017; Mullo-Abdolov, 2017; Protsyuk, 2014b; Protsyuk, 2014c). The software specially developed for the FON project was used (Andruk, 2016a; Andruk 2016b; Kolchinskii, 1977; Pakuliak, 2016).

The programs and applications used to process digitized frames have been described in the studies (Andruk, 2015a; Andruk, 2015b; Andruk, 2017; Protsyuk, 2014a). In order to estimate the astrometric and photometric accuracy of the Epson Perfection V700 Photo scanner, six consecutive scans of the plate representing the Pleiades star cluster (No 17,000; ORWO-ZU21) were processed with the Seven Wide Angle Astrograph at the Astronomical Observatory of Odessa National University with 30-minute exposure and the resulting scan size of $18 \times 24 \mathrm{~cm}$. The scanning was performed in two scanning modes with spatial resolutions of 1,200 and 2,400 dpi, respectively. The computations for all objects recorded on the scanned plate (about 9,100 objects) were carried out as follows. The objects' characteristics, namely Cartesian $\mathrm{X}$ and $\mathrm{Y}$ coordinates and instrumental magnitudes $\mathrm{m}$, averaged with respect to six scans were obtained for each scanning mode separately. A set of diagrams in Fig. 1 presents the following results of scanning at 1,200 dpi. Fig. 1a on the left depicts how the X-coordinates determined from each of six consecutive scans are spread out from the averaged ones (1a, $2 \mathrm{a}, 3 \mathrm{a}, 4 \mathrm{a}, 5 \mathrm{a}$ and $6 \mathrm{a})$. The scatter of the X-coordinates about their means after systematic error reduction is illustrated in the diagrams on the right $(1 \mathrm{~b}, 2 \mathrm{~b}, 3 \mathrm{~b}, 4 \mathrm{~b}, 5 \mathrm{~b}$ and $6 \mathrm{~b}$ ). The standard deviation calculated for one of the differences between the estimated and averaged X-coordinates is indicated for all six scans. The bottom part 

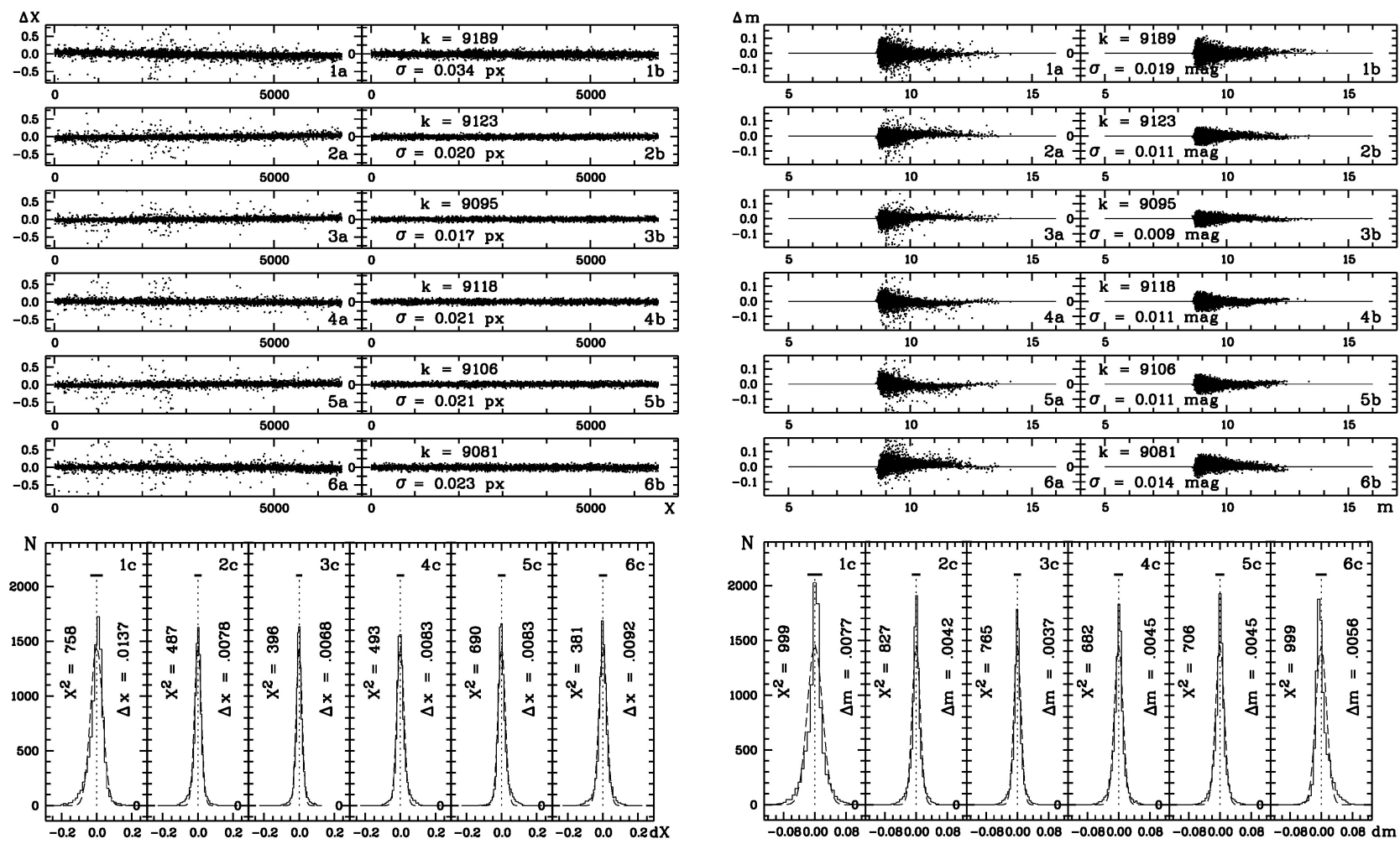

Figure 1a. Scanning at 1200 dpi. The scatter of X-coordinates determined from each of six consecutive scans about the averaged ones.
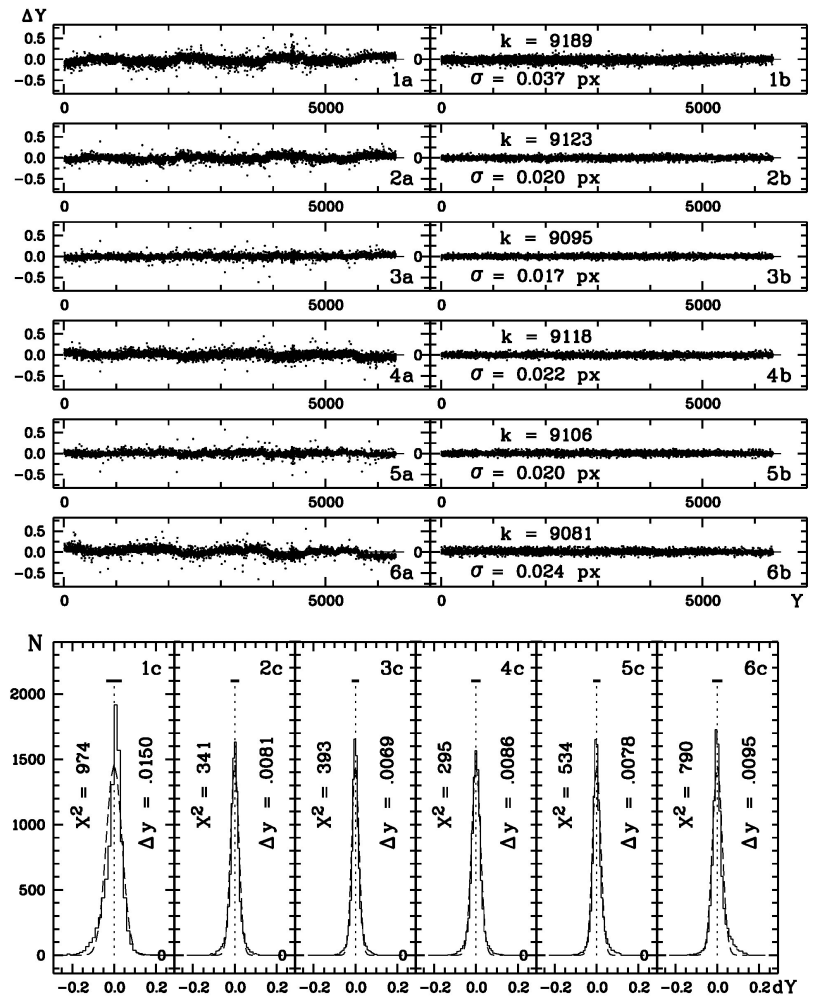

Figure 1b. Scanning at $1200 \mathrm{dpi}$. The scatter of Y-coordinates determined from each of six consecutive scans about the averaged ones.

Figure 1c. Scanning at 1200 dpi. The scatter of instrumental magnitudes $m$ estimated from each of six consecutive scans around the averaged ones.

of the figure shows the frequency distribution for these differences. The differences between the estimated and mean X-coordinates are presented as actual (solid lines) and theoretical (dashed lines) distribution functions over the respective intervals. The interval lengths $\Delta \mathrm{X}$ and $\mathrm{X}^{2}$ values are given on the left and right side of the bottom diagrams, respectively. Likewise, Figs. $1 \mathrm{~b}$ and 1c demonstrate the scatter about the means and its characteristics for Y-coordinates and instrumental magnitudes, respectively. The results of processing of six scans at 2,400 dpi are plotted in a series of diagrams in Fig. 2.

Figs. $1 \mathrm{a}-1 \mathrm{c}$ and $2 \mathrm{a}-2 \mathrm{c}$ present the results obtained for the stars brighter than $\mathrm{B}<13^{\mathrm{m}}$. The magnitude scale was calibrated using the compiled catalogue of photoelectric UBVR stellar magnitudes (Relke, 2015). Thus, the standard deviation (the root-mean-square deviation) of an estimate of the scatter of the Cartesian coordinates is $\sigma_{x y}=0.023 \pm 0.007 \mathrm{px}$ and $\sigma_{x y}=0.082 \pm 0.008 \mathrm{px}$ when scanning at 1,200 dpi and 2,400 dpi, respectively. The standard deviation of the difference between the estimated and averaged instrument magnitudes is $\sigma_{m}=0.013^{\mathrm{m}} \pm 0.004^{\mathrm{m}}$ and $\sigma_{m}=0.042^{\mathrm{m}} \pm 0.003^{\mathrm{m}}$ at 1,20 and 2,400 dpi, respectively.

The above standard deviation is the measure of the scanner's accuracy in the instrumental system. When converting to the equatorial coordinate system and Johnson system, the standard deviation will change by a scale 

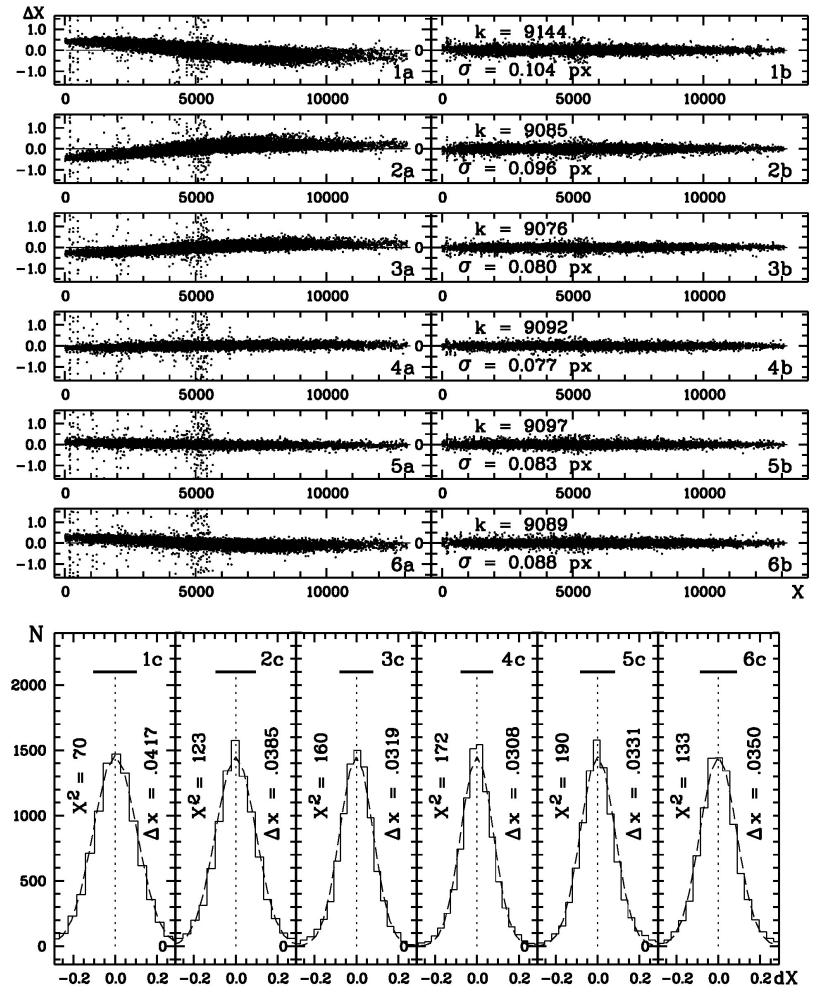

Figure 2a. Scanning at 2400 dpi. The scatter of X-coordinates determined from each of six consecutive scans about the averaged ones.
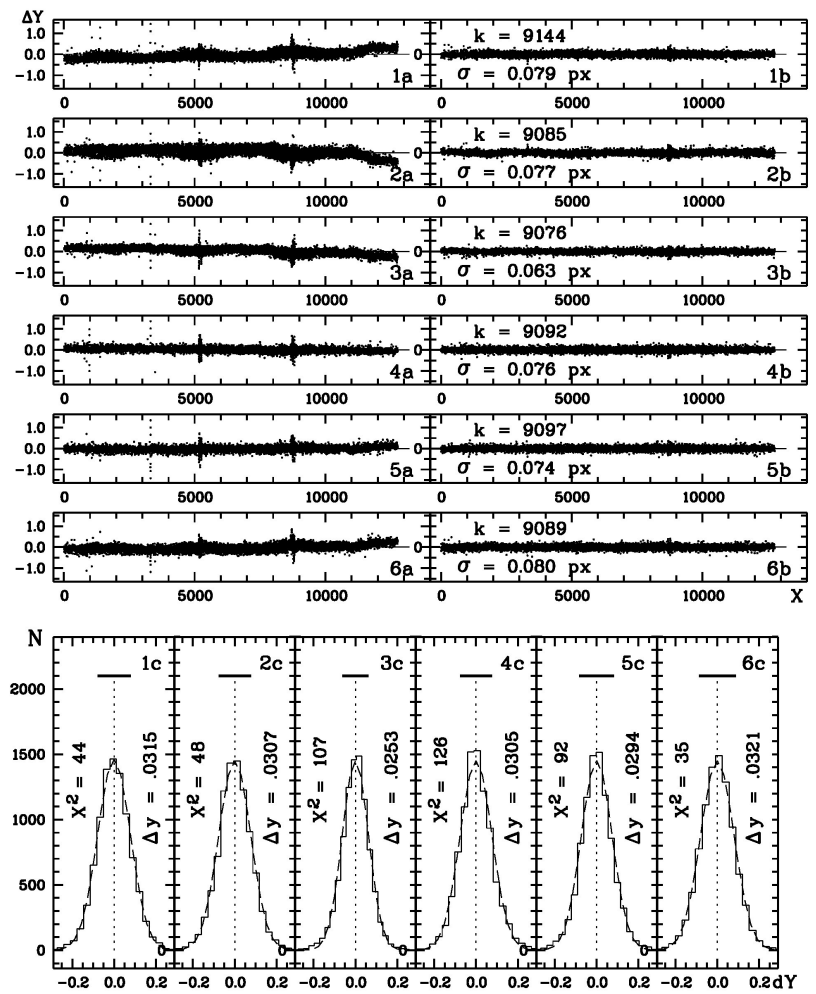

Figure 2b. Scanning at $2400 \mathrm{dpi}$. The scatter of Y-coordinates determined from each of six consecutive scans about the averaged ones.
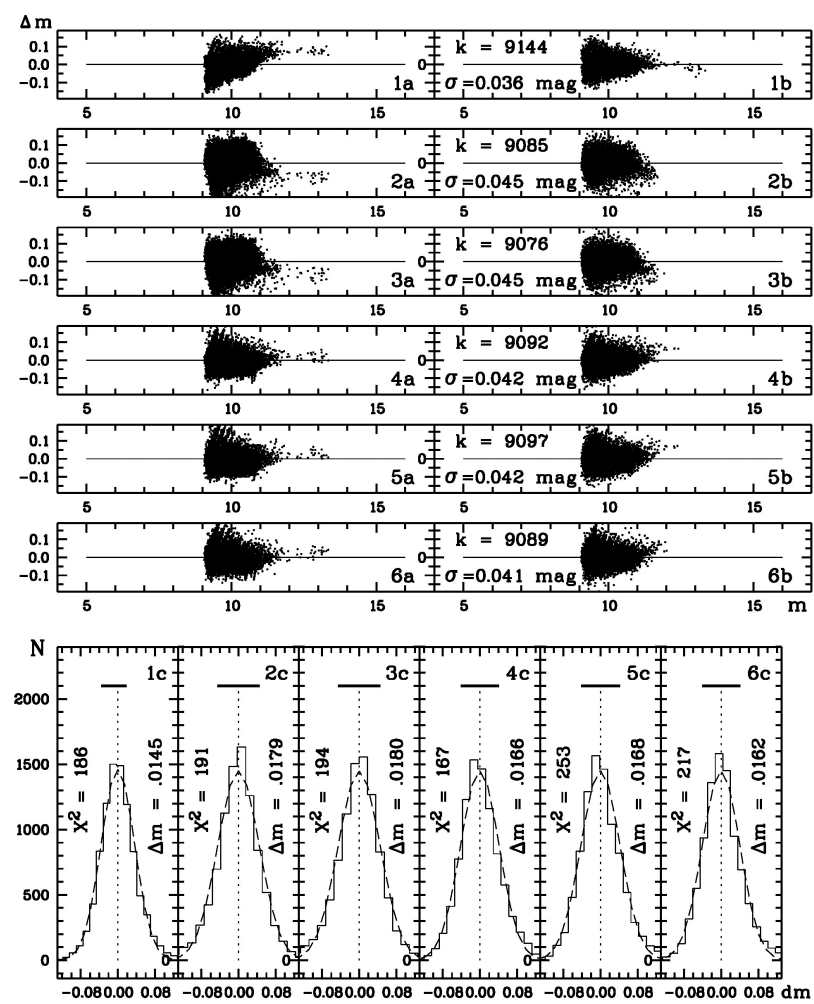

Figure 2c. Scanning at 2400 dpi. The scatter of instrumental magnitudes $m$ estimated from each of six consecutive scans around the averaged ones.

factor for the Cartesian coordinates and by the photo emulsion contrast value for magnitudes.

The authors has drawn conclusion that the Epson Perfection V700 Photo flatbed scanner being used with the specially developed software enables digitising and processing astroplates, as well as obtaining characteristics of objects with an internal accuracy not greater than \pm 0.15 arcseconds and \pm 0.03 magnitude when scanning at 1,200 dpi. When scanning at 2,400 dpi, the internal accuracy is \pm 0.25 arcseconds and \pm 0.1 magnitude for Cartesian coordinates and instrumental magnitudes, respectively.

\section{Conclusion}

The study conducted to specify technical characteristics of processing astroplates digitised with the Epson Perfection V700 Photo scanner has produced the following results. Within the accuracy of estimates for the photographic plates, the Epson scanner is well suited to performing ground-based astrometric and photometric studies. 


\section{References}

Andruk V. M. et al.: 2015, arxiv.org/abs/1512.05535.

Andruk V. M., Pakuliak L. K., Golovnia V. V., et al.: 2015, Odessa Astron. Publ., 28, 192.

Andruk V. M., Golovnia V. V., Ivanov G. A., et al.: 2016 , Kinem. Phys. Cel. Bodies, 32, N1, 38.

Andruk V. M., Pakuliak L. K., Golovnia V. V., et al.: 2016, Kinem. Phys. Cel. Bodies, 32, N5, 260.

Andruk V. M., Pakuliak L. K., Golovnia V. V., et al.: 2017, Science and Innovation, 13a, N1, 17.

Golovnya V., Andruk V., Yatsenko A.: 2010, J. of Phys. Studies, 14, N2, 2902 (in Ukraine).

Eglitis I., Andruk V.: 2017, Open Astronomy, 26, N1, 7.

Kolchinskii I. G., Onegina A. B.: 1977, Astrometry and Astrophysics, N33, 11 (in Ukraine).
Mullo-Abdolov A., Kokhirova G., Relke H. et al.: 2017, Odessa Astron. Publ., 30, this issue

Pakuliak L. K., Andruk V. M., Golovnia V. V., et al.: 2016, Odessa Astron. Publ., 29, N1, 132.

Protsyuk Yu. I., Andruk V. N., Kazantseva L. V.: 2014, Odessa Astron. Publ., 27, N1, 59.

Protsyuk Yu. I., Andruk V. N., Kazantseva L. V., et al.: 2014, Odessa Astron. Publ., 27, N1, P..61.

Protsyuk Yu. I., Kovylianska O. E., Protsyuk S. V., et al.: 2014, Odessa Astron. Publ., 27, N1, 63.

Relke E., Protsyuk Yu.I., Andruk V.M., et al.: 2015, Odessa Astron. Publ., 28, 211.

Vavilova I. B., Pakuliak L. K., Protsyuk Yu. I., et al.: 2011, Kosm. nauka tehnol, 17, N3, p. 74-91 